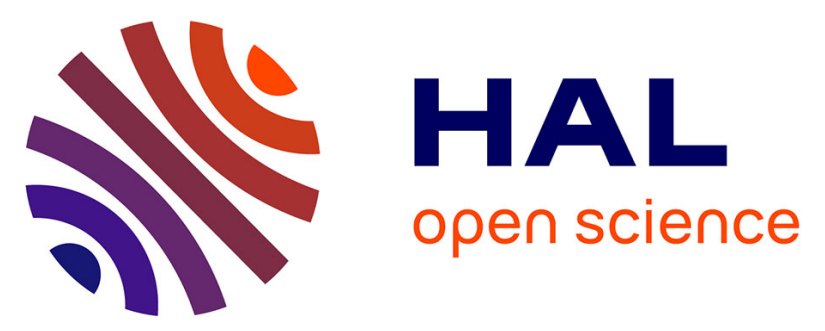

\title{
Thermal phase fluctuations in optically pumped dual-frequency vertical external-cavity surface-emitting lasers for cesium clocks based on coherent population trapping
}

\author{
G Gredat, H Liu, Fabien Bretenaker
}

\section{To cite this version:}

G Gredat, H Liu, Fabien Bretenaker. Thermal phase fluctuations in optically pumped dual-frequency vertical external-cavity surface-emitting lasers for cesium clocks based on coherent population trapping. Journal of Applied Physics, 2019, 126, 10.1063/1.5123444 . hal-02931009

\author{
HAL Id: hal-02931009 \\ https://hal.science/hal-02931009
}

Submitted on 4 Sep 2020

HAL is a multi-disciplinary open access archive for the deposit and dissemination of scientific research documents, whether they are published or not. The documents may come from teaching and research institutions in France or abroad, or from public or private research centers.
L'archive ouverte pluridisciplinaire HAL, est destinée au dépôt et à la diffusion de documents scientifiques de niveau recherche, publiés ou non, émanant des établissements d'enseignement et de recherche français ou étrangers, des laboratoires publics ou privés. 


\title{
Thermal phase fluctuations in optically pumped dual-frequency vertical external-cavity surface-emitting lasers for cesium clocks based on coherent population trapping
}

\author{
Cite as: J. Appl. Phys. 126, 173104 (2019); doi: 10.1063/1.5123444 \\ Submitted: 6 August 2019 - Accepted: 19 October 2019 . \\ Published Online: 6 November 2019 \\ G. Gredat, ${ }^{1}$ (D) H. Liu, ${ }^{1,2, a)}$ and F. Bretenaker ${ }^{1,3, b)}$ (D)

\begin{abstract}
AFFILIATIONS
${ }^{1}$ Laboratoire Aimé Cotton, Université Paris-Sud, ENS Paris-Saclay, CNRS, Université Paris-Saclay, 91405 Orsay, France

${ }^{2}$ Institute of Photonics and Photon Technology, Northwest University, Xi'an 710069, China

${ }^{3}$ Light and Matter Physics Group, Raman Research Institute, Bangalore 560080, India

a)huiliu0629@foxmail.com

b) fabien.bretenaker@u-psud.fr
\end{abstract}

\begin{abstract}
A fully analytical model is established for the thermal fluctuations of the beatnote phase of an optically pumped dual-frequency vertical-external-cavity surface-emitting laser (VECSEL). This model starts with the resolution of the heat equation inside the semiconductor chip structure and follows with the evaluation of the induced thermo-optic phase shift. Both the fluctuations of the heat induced by the optical pumping and the thermodynamic fluctuations at room temperature are considered. On the one hand, the thermal response of the structure is investigated and a significant thermal lens effect caused by the pump is deduced. On the other hand, the power spectral density of the frequency noise is calculated in the presence of diffusion spatial anisotropy. The present model is in very good agreement with the phase noise measured for a dual-frequency VECSEL at $852 \mathrm{~nm}$ for application to metrology and the validity of the usual low-pass filter model is discussed.
\end{abstract}

Published under license by AIP Publishing. https://doi.org/10.1063/1.5123444

\section{INTRODUCTION}

Over the last few decades, the properties of vertical-externalcavity surface-emitting lasers (VECSELs) have raised interest for various applications. Although their miniaturization is still being actively investigated, ${ }^{1}$ VECSELs are rather mechanically compact, which is required for their integration in systems. They can display a large optical output power ${ }^{2}$ and excellent beam quality, which are, for instance, crucial for medical or biological imaging applications. ${ }^{3}$

The most striking feature exhibited by these lasers is their high spectral purity and low intensity noise allowed by their class-A dynamical behavior. This is made possible by their cavity photon lifetime, which is typically one order of magnitude longer than the lifetime of semiconductor carriers, ${ }^{4}$ preventing relaxation oscillations from appearing. This behavior is in sharp contrast with one of the solid-state lasers. The possibility to generate very low noise RF signals resulting from the beatnote between two orthogonally linearly polarized modes ${ }^{5}$ with a dual-frequency VECSEL (DF-VECSEL) is a significant progress for many applications. This dual-frequency operation is obtained by inserting a birefringent crystal inside the laser's external cavity. With very low intensity and phase noises, VECSELs can even be used for metrology applications, for example as local oscillators for ultrastable atomic clocks. $^{6-8}$ The present paper focuses on this latter application, which is related to the ground state hyperfine transition around $9.2 \mathrm{GHz}$ of cesium 133 atoms. Indeed, DF-VECSELs operating at $852 \mathrm{~nm}$ are promising sources to build clocks combining both a good relative frequency stability and compactness. ${ }^{6,9}$ This wavelength of $852 \mathrm{~nm}$ corresponds to the $\mathrm{D}_{2}$ transition of cesium. The two emitted orthogonal linear polarizations of a DF-VECSEL can be used to probe a double lambda system along this line, which 
leads to a coherent-population-trapping (CPT) resonance. Such a scheme has been identified as a good candidate to produce high contrast Ramsey fringes for the clock. ${ }^{10}$ The RF beatnote obtained with the DF-VECSEL should then be locked to the clock transition at $9.2 \mathrm{GHz}$, and the beatnote phase noise properties are intimately linked to the induced clock stability. The stringent requirements of this application in terms of noise is a motivation to reach a deeper understanding of its origin.

In the case of optically pumped VECSELs, the main sources of noise are well identified: (i) the pump power fluctuations propagating through the laser dynamics, (ii) the spontaneous emission contribution, (iii) the technical noises such as mechanical vibrations, and (iv) the thermal noise. Noise investigations in VECSELs have led to a quantitative understanding and description of at least the two first sources of noise. ${ }^{4,9,11-18}$ The description of the two other sources of noise deserves more effort, especially the thermal noise. These fluctuations of the local temperature induce changes of the effective optical length of the laser cavity and thus of the laser phase. They can originate from two mechanisms: (i) the thermal loading induced by the pump diode laser transmitting its power fluctuations and (ii) the thermodynamic fluctuations of the temperature inside the structure resulting from the Boltzmann distribution of random deviation from thermal equilibrium. In previous studies, ${ }^{19-21}$ a simple low-pass filter behavior was assumed for the thermal contribution to the frequency noise spectra in VECSELs. However, defining a single cutoff frequency in a nonambiguous manner does not seem that obvious since different time scales come into the picture. Moreover, a disagreement of this model with experimental data has recently been reported. ${ }^{9}$ In this example, the phase noise that was measured corresponded to the beatnote between the two modes emitted by a DF-VECSEL operating at $852 \mathrm{~nm}$. It was observed that the measured phase-noise spectrum of this beatnote exhibits a $f^{-3}$ slope below $200 \mathrm{kHz}$ instead of the $f^{-4}$ behavior predicted by the usual simple model.

This discrepancy proves that it is necessary to go beyond the simple description of the VECSEL thermal noise in terms of a low-pass filter (LPF). The aim of the present paper is thus to develop a microscopic model of the thermal noise contribution to the DF-VECSEL phase noise at $852 \mathrm{~nm}$. In order to gain insight into the physics, we choose an analytical approach instead of a purely numerical one. Furthermore, the predictions of the model are compared to experimental data.

The paper is organized as follows. First, we describe in Sec. II the details of the VECSEL structure used for cesium CPT clocks and the equations we begin with to describe the heat transfer. The thermo-optic effect is also detailed to link the laser phase noise to the thermal fluctuations. Section III focuses on the response of the structure to the pump thermal load. Such an analysis has been done for an electrically pumped vertical cavity surface-emitting laser (VCSEL). ${ }^{22}$ The difference here comes from the geometry and the nature of the excitation so that the typical response time becomes several orders of magnitude larger. To convey a broader message and to fit our experimental context, we also take into account the possible spatial anisotropy of the heat diffusion. We then obtain the temperature map of the VECSEL structure in the steady-state regime and the associated local change of the refractive index of the structure. The phase change it induces for the laser light during propagation is then described by a lenslike effect. Thanks to the fully analytical treatment of the temperature field, this thermal lens effect induced by the pump can be evaluated. Section IV uses the previously determined thermal response of the structure to calculate the frequency noise induced by the pump intensity fluctuations and the thermodynamic temperature fluctuations as well. Comparison with the experimental beatnote phase noise measurements in a DF-VECSEL operating at $852 \mathrm{~nm}$ is performed in Sec. V. A discussion is then initiated to question the validity of the usual simple low-pass filter model and the role of longitudinal heat diffusion.

\section{EXPERIMENTAL CONTEXT AND FRAMEWORK FOR THERMO-OPTIC EFFECTS}

\section{A. Experimental context}

The optically pumped DF-VECSEL cavity operating at $852 \mathrm{~nm}$ and aimed at cesium CPT clocks application is sketched in Fig. 1(a). The semiconductor chip glued to a Peltier cooler, which is itself bonded to a heat sink, is referred to as a $1 / 2$-VCSEL. This chip contains a Bragg mirror, as can be seen in Fig. 1(b), which forms a linear optical cavity of length $L_{\mathrm{CAV}}$ with the concave output coupler of transmission $T$ and radius of curvature $R_{1}$. A $673 \mathrm{~nm}$ multimode-fibered laser diode is pumping the $1 / 2$-VCSEL. For $P_{\text {opt }}=1 \mathrm{~W}$ of incident optical power and an incidence angle $i_{1}=40^{\circ}$, a non-negligible fraction of the pump is reflected while the other part is absorbed inside the structure. An etalon is inserted for mode stabilization. A $1 \mathrm{~mm}$ thick positive uniaxial birefringent crystal of $\mathrm{YVO}_{4}$, which is cut at $45^{\circ}$ off its optical axis, enables dualfrequency operation of two orthogonal linear polarizations denoted as $x$ and $y$. This crystal indeed creates a spatial walk-off inside the laser cavity between the ordinary and extraordinary rays. A ratio $\eta_{\text {th }} \simeq 20 \%$ of the total incident power is thermally dissipated inside the $1 / 2$-VCSEL. The semiconductor chip structure displays three main regions made of $\mathrm{AlGaAs}$ ternary compounds, as sketched in Fig. 1(b). The Peltier is stabilized at $T_{\text {Peltier }}$ close to room temperature. The active part of the $1 / 2$-VCSEL is located on a substrate made of GaAs with a thickness $L_{\mathrm{SUB}}$. On top of the substrate, a distributed Bragg reflector (DBR) of thickness $e_{\mathrm{DBR}}$ is designed to exhibit a very high reflectivity around $\lambda=852 \mathrm{~nm}$, the central wavelength of the laser. The active medium lies on top of this DBR. It is made of several GaAs quantum wells (QWs) embedded in $\mathrm{Al}_{22 \%} \mathrm{GaAs}$ semiconductor barriers. Each of the quantum wells is located at an antinode of the laser field in a resonant design.

The thermally dissipated power inside the structure is responsible for a frequency noise on each polarization mode because of thermo-optic effects, which are going to be investigated. A beatnote phase noise is thus thermally experienced by the DF-VECSEL. Moreover, in semiconductor lasers, intensity noises are creating phase noises because of the phase-amplitude coupling due to the large Henry factor. However, recently, ${ }^{9}$ an optimized pumping architecture has allowed to reduce this phase-amplitude coupling contribution to the beatnote phase noise down to $-120 \mathrm{dBc} / \mathrm{Hz}$. This beatnote phase noise is reproduced in Fig. 1(c) where the measurements are displayed in green open circles. The theoretical spectrum for the phase-amplitude coupling contribution is plotted as a dashed-dotted magenta line. One can clearly see that this 
(a)

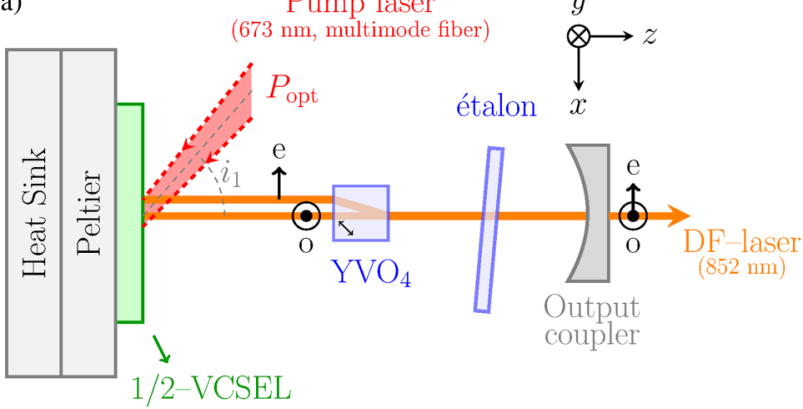

(b)

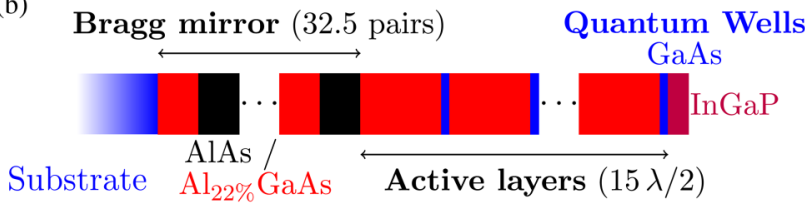

(c)

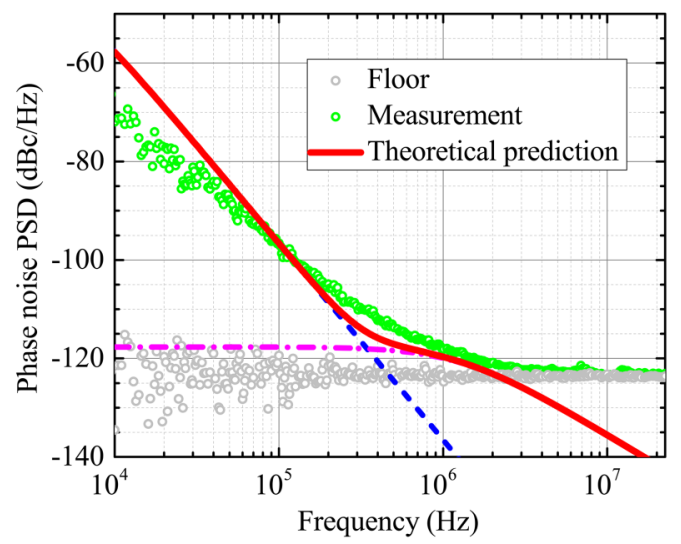

FIG. 1. (a) Dual-frequency VECSEL cavity. $\mathrm{A} \mathrm{YVO}{ }_{4}$ birefringent crystal is inserted to allow dual-frequency operation by creating a walk-off between the ordinary wave (o) and the extraordinary one (e), which are, respectively, $y$ - and $x$-polarized modes. (b) shows the structure of the semiconductor chip used for emission around $852 \mathrm{~nm}$. (c) RF beatnote phase noise power spectral density (PSD) spectra for a dual-frequency VECSEL at $852 \mathrm{~nm}$. ${ }^{9}$ The open gray circles stand for the experimental floor, and the green ones are from measurements. The theoretical spectrum in the solid red line takes into account both the contribution coming from thermal effects in the dashed blue line and the contribution coming from amplitude phase coupling in the dashed-dotted magenta line.

contribution is almost negligible in this result. As a consequence, the only remaining significant contribution to the beatnote phase noise below a few hundreds of kilohertz is coming from the thermal effects. Yet, the low-frequency part of the theoretical spectrum (solid red line) does not fit very well the measurements, which questions the previous model of thermal noise (dashed blue line). A better understanding of the noise coming from thermal effects is, therefore, required for this highly demanding metrology application. In order to establish a more accurate model for the thermal effects contribution to the beatnote phase noise of the
$852 \mathrm{~nm}$ DF-VECSEL, a microscopic approach is implemented. First, for the sake of clarity, a single frequency emitting VECSEL is investigated to model the thermal effects and the induced phase noise. From this work, the case of dual-frequency emission is then deduced in Sec. V.

\section{B. Thermo-optic effect phase shift in the VECSEL}

The single mode laser field emitted at nominal angular frequency $\omega_{l}$ can be written as

$$
E(\mathbf{r}, t)=\mathcal{E}(t) \mathrm{e}^{\mathrm{i}\left[\omega_{l} t+\psi(t)\right]} e(\mathbf{r}),
$$

where $\mathcal{E}(t)$ and $\psi(t)$ are the slowly varying amplitude and phase. The spatial mode profile is denoted as $e(\mathbf{r})$, and its square modulus can be equivalently regarded as the energy density profile of the intracavity standing wave.

Any temperature fluctuation will induce laser phase fluctuations $\delta \psi(t)$. In order to better understand the underlying mechanisms, a description of the effective cavity optical length variation in the presence of a thermal load is required.

The laser single mode volume inside the $1 / 2$-VCSEL is sketched in Fig. 2 (yellow cylinder). The total thickness of the structure is denoted as $L_{\mathrm{SC}}$ and is quite close to $L_{\mathrm{SUB}}$. The spatial laser mode profile $e(\mathbf{r})$ can be separated into its longitudinal $e_{/ /}$and radial $e_{\perp}$ parts, the $z$-axis being the propagation axis. The intensity of the standing wave mode is assumed to be Gaussian in the radial direction. Its waist is denoted as $w_{0}$. The longitudinal part $\left|e_{/ /}(z)\right|^{2}$ is decreasing exponentially into the DBR. Thus, the spatial laser mode profile reads

$$
\begin{aligned}
|e(\mathbf{r})|^{2}= & \mathcal{V}_{\text {mode }}^{-1} \mathrm{e}^{-2\left(\frac{r}{w_{0}}\right)^{2} \cos ^{2}\left(\frac{2 \pi n_{\mathrm{SC}}\left(L_{\mathrm{SC}}-z\right)}{\lambda}\right)} \\
& \times \begin{cases}1 & \text { for } \quad L_{\mathrm{DBR}} \leq z \leq L_{\mathrm{SC}} \\
\exp \left(-\frac{L_{\mathrm{DBR}}-z}{L_{e}}\right) & \text { for } \quad 0 \leq z \leq L_{\mathrm{DBR}},\end{cases}
\end{aligned}
$$

with $\mathcal{V}_{\text {mode }}$ being the mode volume sketched in Fig. 2, used here to normalize the profile to unity, and $r$ is the radius defined as $\sqrt{x^{2}+y^{2}}$. The energy penetration depth within the Bragg reflector $L_{e}$ is found ${ }^{23}$ to verify $L_{e}=\tanh \left(2 \kappa e_{\mathrm{DBR}}\right) / 2 \kappa$, with $\kappa=2 \Delta n / \lambda$ the coupling coefficient, and $\Delta n$ is the superlattice index contrast. Hence, $L_{e}$ appears to be much smaller than $e_{\mathrm{DBR}}$.

We now consider a small local temperature fluctuation $\delta T(\mathbf{r}, t)$. This fluctuation is responsible for a change of the optical length through a local variation of the refractive index and through material thermal expansion. The first effect is referred to as thermorefractive and the second one as thermoelastic. Three different mechanisms of laser phase shift originate from the thermoelastic effect: (i) the substrate thermal expansion creates a reduction of the laser cavity length $L_{\mathrm{CAV}}$ considering that the heat sink and the output coupler are fixed, (ii) the cavity optical length increases with the laser mode volume thermal expansion inside the 1/2-VECSEL, and (iii) the thermal central wavelength shift inside the DBR region creates a laser field phase shift upon reflection. ${ }^{24}$ According to the values of the parameters detailed in the Appendix, we evaluate that these three thermoelastic mechanisms are ranked in the decreasing 


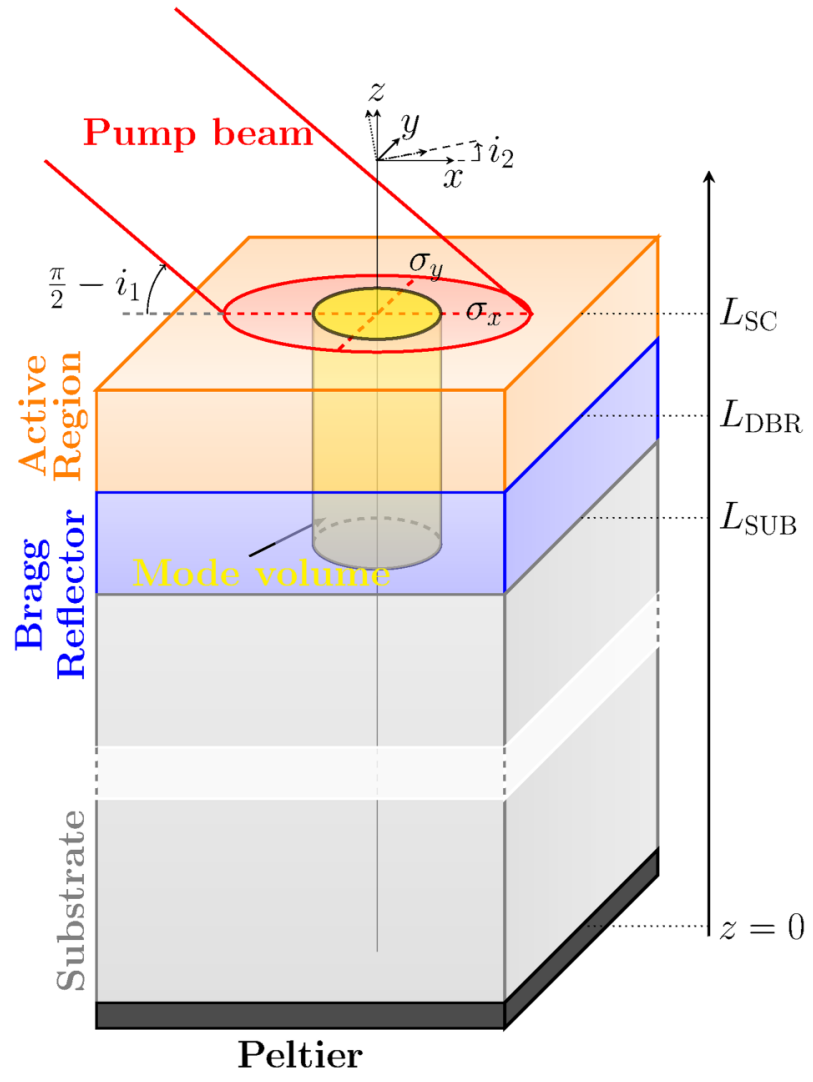

FIG. 2. Simplified cross-section of the 1/2-VCSEL structure with the pumpbeam thermal load. The yellow cylinder sketches the mode volume. The $z$-axis coincides with the optical axis. Its origin is located on the Peltier surface.

order of magnitude. The local strain variations with temperature can be well estimated by the material linear thermal expansion coefficients $\varsigma$. These thermal expansion coefficients are two orders of magnitude lower than the index thermal variation $\partial n_{\mathrm{SC}} / \partial T$. As a result, the dominant laser optical length variation is due to thermorefractive effects. The thermal change of index affects the optical length only in a small portion $L_{\text {mode }}=\mathcal{V}_{\text {mode }} /\left(\pi w_{0}^{2} / 2\right)$ (mode length inside the $1 / 2$-VCSEL) of the total cavity length $L_{\mathrm{CAV}}$. Then, the frequency noise induced by $\delta T(\mathbf{r}, t)$ reads

$$
\delta \dot{\psi}(t)=-q \int_{\mathbb{R}^{3}} \delta T(\mathbf{r}, t)|e(\mathbf{r})|^{2} \mathrm{~d} \mathbf{r},
$$

where

$$
q=\omega_{l} \cdot \frac{L_{\text {mode }}}{L_{\mathrm{CAV}}} \cdot \frac{\partial n_{\mathrm{SC}}}{\partial T}
$$

In this expression, $q$ is the thermorefractive factor. With the parameters of the Appendix, $q$ is found to be of the order of tens of megahertz per Kelvin.
Computing the integrated frequency shift of Eq. (3) requires the knowledge of the temperature distribution inside the structure. To this aim, we use the heat equations to determine this temperature distribution under the influence of two sources of thermal excitation: (i) pump beam absorption and (ii) intrinsic thermodynamic fluctuations at room temperature.

\section{Heat equations}

\section{Pump heating}

The pump beam is provided by a laser diode and is incident on the $(x, y)$ plane with an angle $i_{1}$ with respect to the $z$-axis in the $(x, z)$ incidence plane as shown in Fig. 2. The excitation thus created within the active region makes electrons from the conduction band of the semiconducting layers move until they are captured by a quantum well where they can recombine with holes and emit light. However, this displacement of the carriers comes with energy dissipation and heating. We thus consider that the optical pumping creates a thermal load with a power (expressed in $\mathrm{K} / \mathrm{s}$ ) denoted as $Q(\mathbf{r})$ and switched on at $t=0^{+}$.

The typical dimensions $L_{x}$ and $L_{y}$ of the chip along the $x$ and $y$ directions are about several centimeters, much larger than $L_{\mathrm{SC}}$ (hundreds of micrometers), indicating that our structure can, to some extent, be considered as an infinite plate. The heat equation reads

$$
\left(\partial_{t}-D_{\perp} \nabla_{\perp}^{2}-D_{/ /} \partial_{z}^{2}\right) T(\mathbf{r}, t)=Q(\mathbf{r}), \quad t>0,
$$

with $(x, y, z) \in \mathbb{R}^{2} \times\left[0, L_{S C}\right]$. The coefficients $D_{/ /}$and $D_{\perp}$ are the longitudinal and radial diffusion coefficients, respectively, along the optical axis $z$ and perpendicular to it, i.e., in the $(x, y)$ plane. In such a multilayered structure, some diffusion anisotropy can be expected, meaning that $D_{/ /} \neq D_{\perp}$, especially in the DBR region owing to superlattice effects. ${ }^{25}$ More precisely, the thermal diffusion coefficients in both the longitudinal and perpendicular directions are reduced with respect to the weighted average value of bulk materials. Moreover, this reduction is larger in the perpendicular direction. ${ }^{26}$ Inside the thick substrate of the structure, made of bulk material, thermal diffusion is isotropic with a diffusion coefficient $D$. Instead of using another heat diffusion equation to describe the substrate, we have decided to keep on using Eq. (5) by simply rescaling the thickness of the substrate by a factor $D_{/ /} / D$. Moreover, since the plane perpendicular to $z$ can be well approximated by an infinite plane, we do not need to rescale the transverse directions. This rather crude approximation can be used here because the laser mode does not enter the substrate. An approximate treatment of temperature diffusion in this region will thus not strongly affect the results.

Because of the large value of the refractive index of the semiconductor structure $(\simeq 3.6)$, the refraction angle $i_{2}$ of the pump (see Fig. 2) is of the order of $10^{\circ}$ only. Owing to the small thickness of the active region (a few micrometers) compared to the pump beam diameter (a few tens of micrometers), we thus neglect $i_{2}$ and treat the pump beam as if it were strictly longitudinal. Hence, the pump excitation inside the active region is considered to be centered on the $z$-axis. The beam profile along the perpendicular direction is assumed to be Gaussian, and its widths are given by 
the standard deviations $\sigma_{y}$, which is typically about $10-100 \mu \mathrm{m}$, and $\sigma_{x}=\sigma_{y} / \cos i_{1}$. Since $\sigma_{x} \simeq \sigma_{y} \equiv w_{\mathrm{p}} / 2$, the pump excitation is considered to exhibit cylindrical symmetry around $z$.

The pump power is almost completely absorbed in the QW barriers, with an absorption coefficient $\alpha$. The heat power generated by the pump beam is thus

$$
Q(\mathbf{r})=Q_{0} \cdot \mathrm{e}^{-2\left(\frac{r}{w_{\mathrm{p}}}\right)^{2}} \cdot \mathrm{e}^{-\frac{\left(L_{\mathrm{SC}}-z\right) \alpha}{\cos i_{2}}} .
$$

Only a fraction $\eta_{\text {th }}$ of the incident optical power $P_{\text {opt }}$ is converted into heat, allowing to evaluate $Q_{0}$. Typical values are of the order of a few hundreds of $\mathrm{K} / \mu$ s for $1 \mathrm{~W}$ of incident optical power.

\section{Thermodynamic temperature fluctuations}

A body at ambient temperature $T$ exhibits thermodynamic temperature fluctuations. In a VECSEL structure, the thermo-optic effect will thus lead to fluctuations of the optical thickness and thus to frequency fluctuations that we derive in Sec. IV B. In a homogeneous medium, such temperature fluctuations $\vartheta(t)$ are characterized by their variance $\left\langle\vartheta^{2}\right\rangle=k_{\mathrm{B}} T^{2} / C_{\mathrm{v}} V$, where $k_{\mathrm{B}}$ is Boltzmann's constant, $C_{\mathrm{v}}$ is the specific heat per unit volume, and $\langle\cdot\rangle$ denotes statistical average. ${ }^{27}$ In an inhomogeneous sample, such as the VECSEL chip we consider here, one needs to take diffusion effects into account and thus describe temperature fluctuations $\vartheta(\mathbf{r}, t)$ through a stochastic heat equation

$$
\left(\partial_{t}-D \nabla^{2}\right) \vartheta(\mathbf{r}, t)=\zeta(\mathbf{r}, t), \quad t>0,
$$

where $D$ is an isotropic diffusion coefficient. The Langevin source term $\zeta$ is characterized by the following autocorrelation: ${ }^{28-34}$

$$
\left\langle\zeta(\mathbf{r}, t) \zeta\left(\mathbf{r}^{\prime}, t^{\prime}\right)\right\rangle=\frac{2 D k_{\mathrm{B}} T^{2}}{C_{\mathrm{v}}} \nabla_{\mathbf{r}} \nabla_{\mathbf{r}^{\prime}} \delta\left(\mathbf{r}-\mathbf{r}^{\prime}\right) \delta\left(t-t^{\prime}\right) .
$$

In our case, the thermal properties of the structure are anisotropic. This is taken into account by replacing $D \nabla^{2}$ by the operator $D_{\perp} \nabla_{\perp}^{2}+D_{/ /} \partial_{z}^{2}$ in Eq. (7). The way this anisotropy is taken into account to modify the Langevin force autocorrelation of Eq. (8) is detailed in Sec. IV B.

\section{Boundary conditions}

The Peltier thermoelectric cooler imposes its temperature so that Eqs. (5) and (7) have to be completed with the following boundary conditions:

$$
T(x, y, z=0, t)=T_{\text {Peltier }}=\lim _{x \rightarrow \infty} T(\mathbf{r}, t)=\lim _{y \rightarrow \infty} T(\mathbf{r}, t) .
$$

Furthermore, the thermal conductivity of the chip material is three orders of magnitude larger than the air thermal conductivity under ambient conditions. An isolation cap around the laser cavity also protects the structure from forced air convection. We can, therefore, assume for the system a good enough thermal isolation such that no heat flow is induced from the structure to the air,

$$
\partial_{z} T\left(x, y, z=L_{\mathrm{SC}}, t\right)=0 .
$$

The steady-state and the transient regimes for the thermal response of the structure will now be investigated in order to understand the induced laser phase fluctuations and their spectrum.

\section{THERMAL RESPONSE TO THE PUMP HEATINC}

\section{A. Temperature field inside the structure}

The heat created by the pump is responsible for the local increase of the temperature inside the semiconductor structure. Thanks to the cylindrical symmetry, Hankel transformation at order 0 of Eq. (5) permits to transform the transverse operator $\nabla_{\perp}^{2}$ into an algebraic operator. We denote as $k_{\perp}$ the reciprocal variable of the radial coordinate $r$ and use the following definition of the Hankel transform of a given function $g(r)$ :

$$
\mathrm{HT}[g]\left(k_{\perp}\right) \equiv \int_{\mathbb{R}^{+}} r \mathrm{~d} r g(r) J_{0}\left(k_{\perp} r\right),
$$

with $J_{0}$ being a Bessel function of the first kind. The inverse Hankel transform is denoted as $\mathrm{HT}^{-1}$ and given by

$$
\mathrm{HT}^{-1}[h](r) \equiv \int_{\mathbb{R}^{+}} k_{\perp} \mathrm{d} k_{\perp} h\left(k_{\perp}\right) J_{0}\left(k_{\perp} r\right) .
$$

The Hankel transform of the pump-heating field (6), $\mathrm{HT}[Q]\left(k_{\perp}\right)$, is a simple Gaussian function of standard deviation $2 / w_{\mathrm{p}}$. In the $k_{\perp}$-space, the heat equation becomes a partial differential equation of variables $z$ and $t$. Its solutions are found using a Fourier series expansion along $z$ with separation of variables and taking the boundary conditions (9) and (10) into account. The temperature increase inside the structure $\Delta T(\mathbf{r}, t)=T(\mathbf{r}, t)-T_{\text {Peltier }}$ then reads

$$
\Delta T(\mathbf{r}, t)=\frac{Q_{0}}{2 w_{\mathrm{p}}^{2}} \sum_{n=0}^{\infty} I_{n} \sin \left(\kappa_{n} z\right) \cdot \mathrm{HT}^{-1}\left[\mathcal{F}_{n}\left(k_{\perp}, t\right)\right](r),
$$

with

$$
\mathcal{F}_{n}\left(k_{\perp}, t\right)=\frac{1-\mathrm{e}^{-\left(k_{\perp}^{2} D_{\perp}+\kappa_{n}^{2} D_{/ /}\right) t}}{k_{\perp}^{2} D_{\perp}+\kappa_{n}^{2} D_{/ /}} \mathrm{e}^{-w_{\mathrm{p}}^{2} k_{\perp}^{2} / 8} .
$$

The longitudinal wavenumber $\kappa_{n}=(2 n+1) \pi /\left(2 L_{\mathrm{sc}}\right)$ is coming from the longitudinal Fourier expansion. The coefficients $I_{n}$ of the Fourier expansion of the pump excitation along the $z$-axis are given by

$$
I_{n}=\frac{1}{L_{\mathrm{SC}}} \int_{0}^{L_{\mathrm{SC}}} \mathrm{e}^{-\left(L_{\mathrm{SC}}-z^{\prime}\right) \alpha} \sin \left(\kappa_{n} z^{\prime}\right) \mathrm{d} z^{\prime}
$$

\section{B. Steady-state temperature map}

In the steady-state regime, the term $\mathrm{e}^{-\left(k_{\perp}^{2} D_{\perp}+\kappa_{n}^{2} D_{/ /}\right) t}$ in $\mathcal{F}_{n}\left(k_{\perp}, t\right)$ [see Eq. (14)] vanishes to zero. Unfortunately, the reverse Hankel transform of the remaining term has no analytical 
expression, except along the $z$-axis, where it reads

$$
\Delta T_{\mathrm{ss}}(r=0, z)=\frac{Q_{0} w_{\mathrm{p}}^{2}}{4 D_{\perp}} \sum_{n=0}^{\infty} \mathrm{e}^{\frac{\kappa_{n}^{2} w_{\mathrm{p}}^{2} D_{/ /}}{8 D_{\perp}}} I_{n} \cdot \sin \left(\kappa_{n} z\right) \cdot \mathrm{E}_{1}\left(\frac{\kappa_{n}^{2} w_{\mathrm{p}}^{2}}{8} \frac{D_{/ /}}{D_{\perp}}\right),
$$

where $\mathrm{E}_{1}(x)=\int_{x}^{+\infty} \frac{\exp (-s)}{s} \mathrm{~d} s$ is the so-called exponential integral special function.

The temperature map $\Delta T_{\mathrm{ss}}(r, z)$ shown in Fig. 3 has been computed from Eq. (13) for the steady-state regime using an efficient Hankel transformation numerical algorithm based on matrix products. ${ }^{35}$ The values of the parameters are detailed in the Appendix. One notices the hot spot at the surface of the structure coming from the optical pumping. It exhibits a maximum temperature increase of $86 \mathrm{~K}$. The full-width at half-maximum (FWHM) of the temperature distribution at the surface (red line at the right of Fig. 3) is found to be equal to $30 \mu \mathrm{m}$, which is close to the FWHM $w_{\mathrm{p}} \sqrt{2 \ln (2)}$ of the pump beam. A comparison of the longitudinal and transverse widths of the isotherms witnesses the anisotropy of the diffusion coefficients mentioned above. The longitudinal temperature profiles at the bottom of Fig. 3 are the result of both thermal diffusion and pump absorption. Both longitudinal and radial profiles clearly satisfy boundary conditions (9) and (10). Moreover, the evolution of the radial temperature profile with depth (magenta line vs red line) also evidences the transverse heat flow.

In Sec. III C, we use this temperature map inside the semiconductor chip to deduce the spatial distribution of the refractive index change induced by the thermo-optic effect.

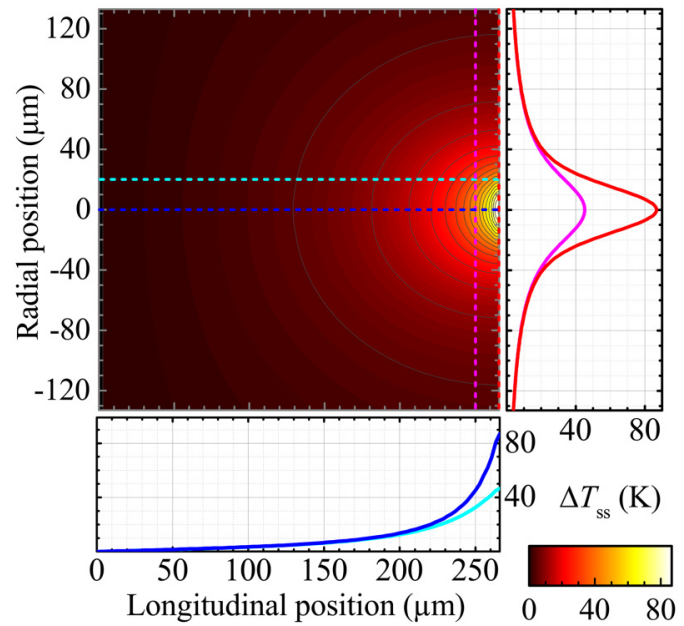

FIG. 3. Calculated steady-state spatial distribution of the temperature increase in the semiconductor structure. The longitudinal (respectively radial) temperature profiles at the bottom (respectively at the right side) of the map are plotted along the blue and cyan (respectively red and magenta) dotted lines. The gray lines on the map are the isotherms.

\section{Thermal lens effect}

In the steady-state regime, the temperature map of Fig. 3 can be used to calculate the thermal phase shift accumulated by the laser field after one round-trip inside the cavity at radial position $r,{ }^{36}$

$$
\Delta \psi(r)=-\frac{2 \pi}{\lambda} \int_{0}^{L_{\mathrm{SC}}} \Delta T_{\mathrm{sS}}(r, z) \cdot \frac{\mathrm{d} n_{\mathrm{SC}}}{\mathrm{d} T}|e(z)|^{2} \mathrm{~d} z .
$$

This phase shift can be compared with the one created by a lens of focal length $f$ and given by $\Delta \psi_{f}(r)=\pi r^{2} / \lambda f$. A Taylor expansion of $\Delta \psi(r)$ in powers of $r$ permits to identify the $r^{2}$ component as a lenslike effect and to calculate the associated focal length. ${ }^{36,37}$ This is performed by expanding $J_{0}\left(k_{\perp} r\right)$ as $1-\left(k_{\perp} r / 2\right)^{2}$ in the inverse Hankel transform (12) of $\mathcal{F}_{n}$ in Eq. (13) for $t \rightarrow \infty$. After identification of the $r^{2}$ term and integration along $k_{\perp}$, the focal length of the thermal lenslike effect is found to be

$$
\begin{aligned}
& f^{-1}=\frac{\partial n_{\mathrm{SC}}}{\partial T} \sum_{n=0}^{\infty} \int_{0}^{L_{\mathrm{SC}}}|e(z)|^{2} \sin \left(\kappa_{n} z\right) \mathrm{d} z \cdot \frac{Q_{0} I_{n}}{D_{\perp}} \\
& \times\left[1-\frac{w_{\mathrm{p}}^{2} \kappa_{n}^{2}}{8} \frac{D_{/ /}}{D_{\perp}} \mathrm{e}^{\frac{w_{\mathrm{p}}^{2} \kappa_{n}^{2} D_{/ /}}{8 D_{\perp}}} \mathrm{E}_{1}\left(\frac{w_{\mathrm{p}}^{2} \kappa_{n}^{2}}{8} \frac{D_{/ /}}{D_{\perp}}\right)\right] .
\end{aligned}
$$

With the values of the parameters given in the Appendix for a $852 \mathrm{~nm}$ optically pumped VECSEL, this focal length is found to be as short as a few millimeters. This lens effect is dramatic because the strong transverse gradient of temperature coming from the hot spot is inducing a strong refractive index variation. A thermal lens associated with the DBR plane mirror is equivalent to a concave mirror with radius of curvature $R_{2}=2 f$. The "new" cavity to be considered once the thermal steadystate is reached is described by the two " $g$ parameters": $g_{1,2}=1-L_{\text {cav }} / R_{1,2}$. The beam radii on the two mirrors can be evaluated using these parameters. ${ }^{37}$ For a cold cavity, when $R_{2} \rightarrow \infty$, the intracavity laser waist is located onto the semiconductor chip. However, after thermal equilibrium is reached, the position of the waist is shifted and the beam radius on the active surface is found to be $w_{2}=24 \mu \mathrm{m}$ with the parameters of the Appendix. In the following, we thus replace $w_{0}$ by $w_{2}$ in Eqs. (2) and (4).

\section{Transient thermal response}

The values of the parameters given in the Appendix lead to the following orders of magnitude for the different longitudinal and transverse time scales involved in our system: $\left(L_{\mathrm{SC}}-L_{\mathrm{DBR}}\right)^{2} / D_{/ /} \simeq 1 \mu \mathrm{s}$, $w_{0}^{2} / D_{\perp} \simeq w_{\mathrm{p}}^{2} / D_{\perp} \simeq 10-100 \mu \mathrm{s}, L_{\mathrm{SC}}^{2} / D_{/ /} \simeq 1 \mathrm{~ms}$, and $L_{x, y}^{2} / D_{\perp} \simeq 1 \mathrm{~s}$, leading to the following hierarchy of time scales:

$$
\frac{\left(L_{\mathrm{SC}}-L_{\mathrm{DBR}}\right)^{2}}{D_{/ /}} \ll \frac{w_{0}^{2}}{D_{\perp}} \simeq \frac{w_{\mathrm{p}}^{2}}{D_{\perp}} \ll \frac{L_{\mathrm{SC}}^{2}}{D_{/ /}} \frac{L_{x, y}^{2}}{D_{\perp}} .
$$

Defining a single thermal response time for the system is thus difficult. If we focus on longitudinal diffusion only, Eq. (13) provides 
an analytic expression of the temperature field along the $z$-axis,

$$
\begin{aligned}
\Delta T(r=0, z, t)= & \frac{Q_{0} w_{\mathrm{p}}^{2}}{4 D_{\perp}} \sum_{n=0}^{\infty} \mathrm{e}^{\frac{\kappa_{n}^{2} w_{\mathrm{p}}^{2} D_{/ /}}{8} D_{\perp}} I_{n} \sin \left(\kappa_{n} z\right) \\
& \times\left\{\mathrm{E}_{1}\left(\frac{\kappa_{n}^{2} w_{\mathrm{p}}^{2}}{8} \frac{D_{/ /}}{D_{\perp}}\right)-\mathrm{E}_{1}\left[\frac{\kappa_{n}^{2}}{2} \frac{D_{/ /}}{D_{\perp}}\left(\frac{w_{\mathrm{p}}^{2}}{4}+2 D_{\perp} t\right)\right]\right\} .
\end{aligned}
$$

The time evolution of this longitudinal temperature field (20) is represented in Fig. 4(a) for several values of the depth $z$, ranging from the top of the structure to the Peltier cooler. The typical time to reach steady-state is of the order of a few milliseconds. More precisely, this longitudinal time scale is given by $L_{\mathrm{SC}}^{2} / D_{/ /} \simeq 3 \mathrm{~ms}$. The maximum
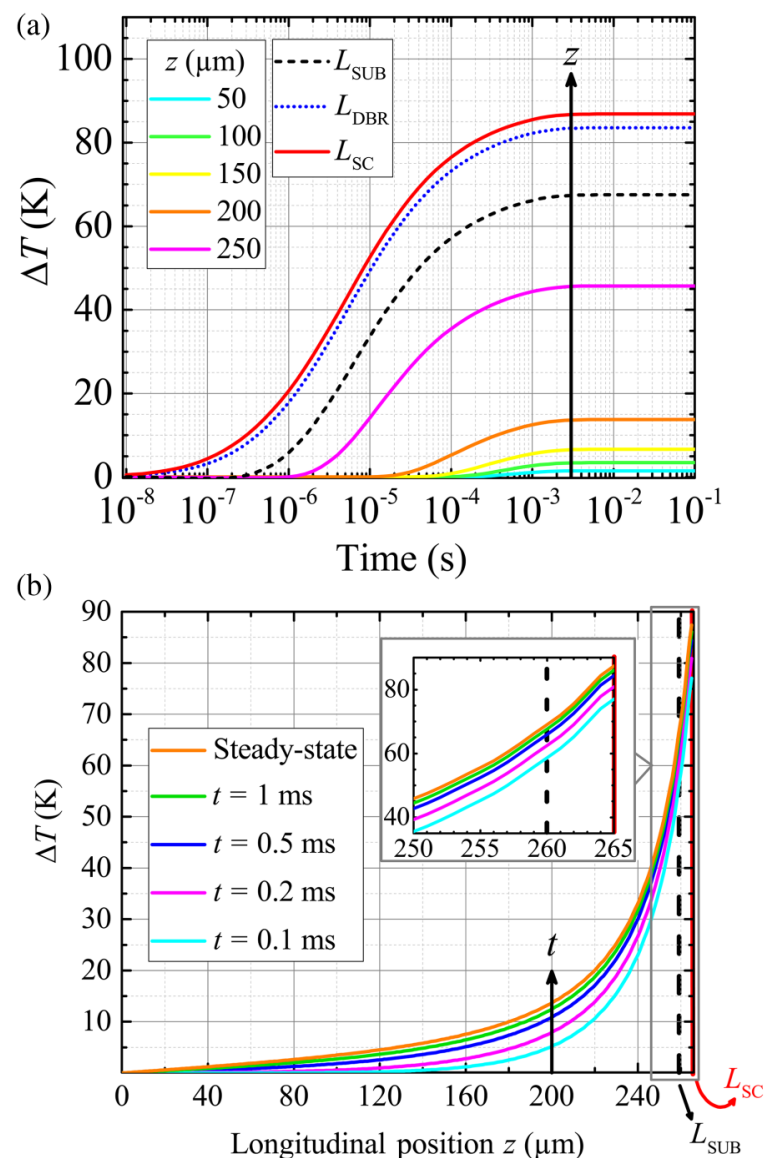

FIG. 4. Evolution of the temperature increase $\Delta T$ induced by pump heating for $x=y=0$. The pump is switched on at $t=0$. (a) Evolution of $\Delta T$ vs $t$ for different values of $z$. (b) Evolution of $\Delta T$ vs $z$ for different values of $t$. The orange line is computed with the steady-state expression given in (16). The inset is a zoom around the active part of the structure. The dashed black line corresponds to the limit between the substrate and the Bragg mirror, and the solid red one corresponds to the surface of the structure. value of the temperature increase is located at the surface and reaches about $86 \mathrm{~K}$, as already seen before. The typical time for the temperature variation to reach half of its steady-state value decreases when $z$ increases because of the delay for longitudinal propagation of heat that evolves like $\left(L_{\mathrm{SC}}-z\right)^{2} / D_{/ /}$. It saturates around $w_{\mathrm{p}}^{2} / 2 D_{\perp}$ as a consequence of radial heat diffusion. The argument of the exponential integral function in the longitudinal temperature profile of Eq. (20) evidences the intricate mixture of longitudinal and transverse diffusions. Indeed, for each component of the longitudinal Fourier series expansion, it depends on the ratio between the transverse and longitudinal diffusions.

The longitudinal temperature profile at several instants $t$ after the pump has been switched on is shown in Fig. 4(b), based on Eqs. (20) and (16). It corroborates the previous time scale of a few milliseconds to reach steady-state. It also confirms that the steadystate temperatures are reached sooner near the surface than deeper inside the structure, as a consequence of the propagation delay mentioned above.

Let us now focus on the volume occupied by the laser mode in the structure. The penetration depth of the laser field inside the structure is of the order $L_{\mathrm{SC}}-L_{\mathrm{SUB}} \simeq 6 \mu \mathrm{m}$, corresponding to a thermal response time of the order of $1 \mu \mathrm{s}$, as can be seen in Fig. 4(a). Besides, the transverse heat diffusion time over the transverse laser mode section is of the order of $10 \mu \mathrm{s}$. These two characteristic response times are the ones that will govern the noise spectra that will be investigated below.

\section{FREQUENCY NOISE INDUCED BY THERMAL FLUCTUATIONS}

\section{A. Contribution of the pump noise}

The pump laser exhibits intensity fluctuations that are described by its relative intensity noise spectrum $\operatorname{RIN}_{p}(\omega)$. To take these fluctuations into account, we replace the quantity $Q_{0}$ of Eq. (6) by $Q_{0}+\delta Q(t)$, where $\delta Q(t)$ is proportional to the pump laser intensity fluctuations. This in turn will be used to deduce the temperature fluctuations $\delta T(\mathbf{r}, t)$ from Eq. (13) using a Green function approach described below. Then, the temperature fluctuations are turned into laser frequency fluctuations through the thermo-optic effect described in Subsection II B.

\section{Calculation of the Green function}

To deduce the evolution of the temperature under the influence of the time varying pump power, we derive the Green function $G_{T}(\mathbf{r}, t)$ obeying the heat equation (5) in which the time dependence is replaced by a Dirac function,

$$
\left(\partial_{t}-D_{\perp} \nabla_{\perp}^{2}-D_{/ /} \partial_{z}^{2}\right) G_{T}(\mathbf{r}, t)=\delta(t) \frac{Q(\mathbf{r})}{Q_{0}} .
$$

The spatial profile $Q(\mathbf{r}) / Q_{0}$ of the heat source is given by Eq. (6). The solution $G_{T}(\mathbf{r}, t)$ is found using the same resolution steps as in Subsection III A, leading to

$$
\widetilde{G}_{T}(\mathbf{r}, \omega)=\frac{w_{\mathrm{p}}^{2}}{2} \sum_{n=0}^{\infty} I_{n} \sin \left(\kappa_{n} z\right) \cdot \mathrm{HT}^{-1}\left[\mathcal{G}_{n}\left(k_{\perp}, \omega\right)\right](r),
$$


with

$$
\mathcal{G}_{n}\left(k_{\perp}, \omega\right)=\frac{\mathrm{e}^{-w_{\mathrm{p}}^{2} k_{\perp}^{2} / 8}}{\mathrm{i} \omega+\left(k_{\perp}^{2} D_{\perp}+\kappa_{n}^{2} D_{/ /}\right)},
$$

and where $\sim$ denotes the time Fourier transformed function at angular frequency $\omega$.

The laser instantaneous frequency fluctuations $\delta \dot{\psi}(t)$ are then obtained by writing the linear response of the system to the fluctuations $\delta Q(t)$ of the heating through

$$
\delta \dot{\psi}(t)=\int_{\mathbb{R}} \mathrm{d} \tau G_{\dot{\psi}}(t-\tau) \cdot \delta Q(\tau) .
$$

The thermal susceptibility $G_{\dot{\psi}}$ is related to the thermal Green function $G_{T}$ of Eq. (21) through the thermo-optic effect described by Eq. (3),

$$
\widetilde{G_{\dot{\psi}}}(\omega)=-q \int_{\mathbb{R}^{3}} \widetilde{G}_{T}(\mathbf{r}, \omega) \cdot|e(\mathbf{r})|^{2} \mathrm{~d} \mathbf{r} .
$$

This integral can be calculated using the Parseval-Plancherel theorem for Hankel transforms,

$$
\begin{aligned}
\int_{\mathbb{R}^{+}} & r \mathrm{~d} r \mathrm{HT}^{-1}\left[\mathcal{G}_{n}\left(k_{\perp}, \omega\right)\right](r) \cdot \mathrm{e}^{-2\left(\frac{r}{w_{2}}\right)^{2}} \\
= & \int_{\mathbb{R}^{+}} k_{\perp} \mathrm{d} k_{\perp} \mathcal{G}_{n}\left(k_{\perp}, \omega\right) \cdot \frac{w_{2}^{2}}{4} \cdot \mathrm{e}^{-\frac{k_{\perp}^{2} w_{2}^{2}}{8}},
\end{aligned}
$$

leading finally to

$$
\begin{aligned}
\widetilde{G_{\dot{\psi}}}(\omega)= & -q \cdot \sum_{n=0}^{\infty} \int_{0}^{L_{\mathrm{SC}}}\left|e_{/ /}(z)\right|^{2} \sin \left(\kappa_{n} z\right) \mathrm{d} z \\
& \times \frac{I_{n} w_{\mathrm{p}}^{2} \pi w_{2}^{2}}{8 D_{\perp} \mathcal{V}_{\text {mode }}} \cdot \exp \left(\frac{a_{n}+\mathrm{i} \omega \tau_{c}}{2}\right) \cdot \mathrm{E}_{1}\left(\frac{a_{n}+\mathrm{i} \omega \tau_{c}}{2}\right),
\end{aligned}
$$

where the longitudinal-to-transverse diffusion ratio $a_{n}$ and the typical transverse diffusion time $\tau_{c}$ over the laser and pump mode radii are given by

$$
\left\{\begin{array}{l}
a_{n}=\frac{\kappa_{n}^{2}}{4} \frac{D_{/ /}}{D_{\perp}}\left(w_{2}^{2}+w_{\mathrm{p}}^{2}\right), \\
\tau_{c}=\frac{w_{2}^{2}+w_{\mathrm{p}}^{2}}{4 D_{\perp}} .
\end{array}\right.
$$

\section{Frequency noise power spectral density}

The linear response of the laser frequency to the heat source fluctuations [see Eq. (24)] leads to the following relation between the power spectral density $S_{\dot{\psi}}$ of the laser frequency noise and the pump laser relative intensity noise:

$$
S_{\dot{\psi}}(\omega)=Q_{0}^{2} \cdot \operatorname{RIN}_{\mathrm{p}}(\omega) \times\left|\widetilde{G_{\dot{\psi}}}(\omega)\right|^{2} .
$$

As a result, the frequency noise PSD reads

$$
S_{\dot{\psi}}(\omega)=\operatorname{RIN}_{\mathrm{p}} \cdot\left(\frac{q Q_{0} \pi w_{2}^{2} w_{\mathrm{p}}^{2} L_{\mathrm{SC}}}{8 D_{\perp} \mathcal{V}_{\text {mode }}}\right)^{2} \cdot\left|\sum_{n=0}^{\infty} \mathcal{N}_{n} \mathcal{A}_{n}(\omega)\right|^{2},
$$

where the longitudinal Fourier series components of the spectral content and their weights are

$$
\left\{\begin{array}{l}
\mathcal{A}_{n}(\omega)=\exp \left(\frac{a_{n}+\mathrm{i} \omega \tau_{c}}{2}\right) \cdot \mathrm{E}_{1}\left(\frac{a_{n}+\mathrm{i} \omega \tau_{c}}{2}\right) \\
\mathcal{N}_{n}=\frac{I_{n}}{L_{\mathrm{SC}}} \int_{0}^{L_{\mathrm{SC}}}\left|e_{/ /}(z)\right|^{2} \sin \left(\kappa_{n} z\right) \mathrm{d} z
\end{array}\right.
$$

The frequency noise spectrum given by Eq. (30) is plotted in Fig. 5, with the parameters summarized in the Appendix. The convergence of the infinite series contained in Eq. (30) is illustrated in the inset. Although the number of terms $n_{\max }$ for the summation to reach a satisfactory precision increases with the noise frequency, only 50 terms are sufficient for a $1 \mathrm{MHz}$ bandwidth. In our experiments, the pump laser has a constant relative intensity noise RIN $_{p}$ over a $10 \mathrm{MHz}$ bandwidth. For this reason, we take a white pump intensity noise. The frequency noise spectrum of Fig. 5 exhibits a $f^{0}$ asymptotic behavior at low frequencies and a $f^{-2}$ slope at high frequencies. The transition between these two behaviors is, however, much smoother than for a simple first-order low-pass filter, and this feature gets more pronounced when the number of Fourier components accounting for longitudinal diffusion is increased. This smooth transition is relatively close to a Flicker $1 / f$ frequency noise in the range going from $1 \mathrm{kHz}$ to $100 \mathrm{kHz}$.

Before comparing these predictions with the experimental phase-noise measurements, the other source of random heat fluctuations at room temperature deserves a similar treatment.

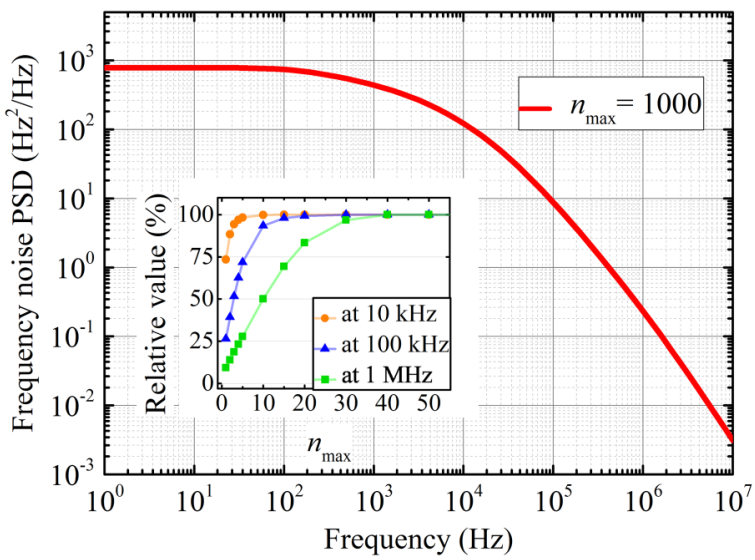

FIG. 5. Optical frequency noise PSD induced by the pump intensity noise. The convergence of the infinite series of its Fourier terms given in Eq. (30) is displayed in the inset for different frequencies, as a function of the maximum value $n_{\max }$ of $n$. Only a few tens of terms are sufficient. The spectrum in the solid red line is plotted with $10^{3}$ terms. 


\section{B. Contribution of the thermodynamic fluctuations}

The thermodynamic temperature fluctuations in the 1/2-VCSEL structure are also responsible of laser frequency fluctuations through the thermo-optic effect of Eq. (3). In this section, we derive an analytical expression for this contribution to the laser frequency noise, based on the stochastic heat equation (7) and the Langevin field correlations of Eq. (8).

Similar studies have been performed on distributed feedback fiber lasers ${ }^{32}$ or (GaAs- and InP-based) VCSELs, ${ }^{31}$ using an infinite plate geometry approximation leading to an analytical result for the phase autocorrelation function. The latter example is quite close to our present problem, but no fully analytical expression of the frequency noise PSD was given, although the Fourier transform of the autocorrelation function was numerically obtained. Further investigations are needed here, especially to take into account the anisotropy between the longitudinal and the transverse diffusions. Note also that the absence of external cavities and the small volume of VCSELs leads to much larger values of the thermo-optic factor, typically of the order of tens of $\mathrm{GHz} / \mathrm{K}$, which makes them more sensitive to thermal fluctuations. One should also notice that their integrated cavity mirrors lead to a different energy profile of the laser mode inside the semiconductor chip.

\section{Spectrum of the thermodynamic temperature fluctuations}

In order to derive the correlation spectrum of the thermodynamic local temperature fluctuations in the infinite plate geometry described in Sec. II, and taking the anisotropy of the thermal diffusion into account, we perform a spatial Hankel transform and a time to frequency Fourier transform of $\zeta(\mathbf{r}, t)$ and $\zeta\left(\mathbf{r}^{\prime}, t^{\prime}\right)$ in Eq. (8). We then attribute different diffusion coefficients $D_{\perp}$ and $D_{/ /}$ to diffusion along the variables $k_{\perp}$ and $z$, respectively, leading to

$$
\begin{aligned}
& \left\langle\mathrm{HT}[\widetilde{\zeta}]\left(k_{\perp}, z, \omega\right) \mathrm{HT}\left[\widetilde{\zeta}^{*}\right]\left(k_{\perp}^{\prime}, z^{\prime}, \omega^{\prime}\right)\right\rangle \\
& \quad=\frac{2 k_{\mathrm{B}} T^{2}}{C_{v}}\left(D_{\perp} k_{\perp}^{2}-D_{/ /} \partial_{z}^{2}\right) \cdot \delta(z-z \prime) \frac{\delta\left(k_{\perp}-k_{\perp}^{\prime}\right)}{k_{\perp}} \cdot \delta\left(\omega-\omega^{\prime}\right) .
\end{aligned}
$$

This Langevin force autocorrelation is then used to find the temperature fluctuations by solving Eq. (7) along the lines of the longitudinal Fourier series expansion of Sec. III, together with boundary conditions (9) and (10), leading to

$$
\begin{aligned}
\langle\mathrm{HT} & {\left.[\widetilde{\vartheta}]\left(k_{\perp}, z, \omega\right) \mathrm{HT}\left[\widetilde{\vartheta}^{*}\right]\left(k_{\perp}^{\prime}, z^{\prime}, \omega^{\prime}\right)\right\rangle } \\
= & \frac{4 k_{\mathrm{B}} T^{2} \delta\left(\omega-\omega^{\prime}\right)}{C_{v} L_{\mathrm{sc}}} \sum_{n=0}^{\infty} \frac{\sin \left(\kappa_{n} z\right) \sin \left(\kappa_{n} z^{\prime}\right)}{\left(D_{\perp} k_{\perp}^{2}+D_{/ /} \kappa_{n}^{2}-\mathrm{i} \omega\right)} \\
& \times \frac{\left(D_{\perp} k_{\perp}^{2}+D_{/ /} \kappa_{n}^{2}\right) \delta\left(k_{\perp}-k_{\perp}^{\prime}\right) / k_{\perp}}{\left(D_{\perp}{k_{\perp}^{\prime}}^{2}+D_{/ /} \kappa_{n}^{2}+\mathrm{i} \omega^{\prime}\right)} .
\end{aligned}
$$

\section{Frequency noise power spectral density}

The laser frequency noise induced by these thermodynamic temperature fluctuations is obtained thanks to the thermo- optic effect of Subsection II B, leading to the following spectrum:

$$
\begin{aligned}
S_{\dot{\psi}}^{\text {Thermo }}(\omega)= & q^{2} \int_{\mathbb{R}^{6}}\left\langle\widetilde{\boldsymbol{\vartheta}}(\mathbf{r}, \omega) \cdot{\widetilde{\vartheta^{*}}}\left(\mathbf{r}^{\prime}, \omega\right)\right\rangle \\
& \times|e(\mathbf{r})|^{2} \cdot\left|e\left(\mathbf{r}^{\prime}\right)\right|^{2} \mathrm{~d} \mathbf{r} \mathrm{d} \mathbf{r}^{\prime} .
\end{aligned}
$$

Injecting Eq. (33) into Eq. (34) and using the ParsevalPlancherel theorem in Hankel domain like in Eq. (26) leads to

$$
\begin{aligned}
S_{\dot{\psi}}^{\text {Thermo }}(\omega)= & \frac{q^{2} \pi^{2} w_{2}^{4} k_{\mathrm{B}} T^{2}}{C_{v} \mathcal{V}_{\text {mode }}^{2} L_{\mathrm{SC}} D_{\perp}} \\
& \times \sum_{n=0}^{\infty}\left(\int_{0}^{L_{\mathrm{SC}}} \mathrm{d} z \sin \left(\kappa_{n} z\right)\left|e_{/ /}(z)\right|^{2}\right)^{2} \\
& \times \operatorname{Re}\left[\exp \left(b_{n}+\mathrm{i} \omega \tau\right) \cdot \mathrm{E}_{1}\left(b_{n}+\mathrm{i} \omega \tau\right)\right],
\end{aligned}
$$

where the longitudinal-to-transverse diffusion ratios $b_{n}$ and typical transverse diffusion time $\tau$ over the mode radius are given by

$$
\left\{\begin{array}{c}
b_{n}=\frac{\kappa_{n}^{2} w_{2}^{2}}{4} \frac{D_{/ /}}{D_{\perp},} \\
\tau=w_{2}^{2} /\left(4 D_{\perp}\right) .
\end{array}\right.
$$

The expression found in Eq. (35) is consistent with the previous ones obtained in the context of either distributed feedback fiber lasers ${ }^{32}$ or VCSELs, ${ }^{31}$ but which were restricted to the case of isotropic heat diffusion $\left(D_{\perp}=D_{/ /}\right)$. Figure 6 shows the corresponding frequency noise PSD plotted with the parameters of the Appendix. The convergence of the infinite sum in Eq. (35) is reached for a number of terms equal to $n_{\max }=60$ for a bandwidth of $1 \mathrm{MHz}$, as shown in the inset of Fig. 6. Like in the case of Fig. 5,

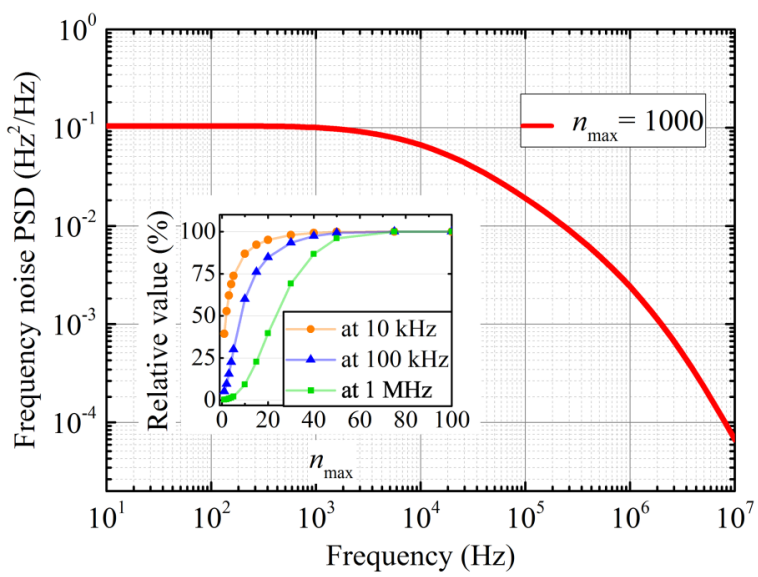

FIG. 6. Optical frequency noise PSD spectrum induced by the thermodynamic fluctuations at room temperature. The convergence of its Fourier series definition given in Eq. (35) is displayed in the inset plot for different frequencies. Only a few tens of terms are required for the longitudinal Fourier series of final index denoted as $n_{\max }$. The spectrum in the solid red line is plotted with $10^{3}$ terms. 
the frequency noise spectrum is white at low frequencies and exhibits a $f^{-2}$ asymptotic behavior beyond a few megahertz, with a smooth transition between $1 \mathrm{kHz}$ and several $100 \mathrm{kHz}$.

\section{COMPARISON WITH THE EXPERIMENTAL BEATNOTE PHASE NOISE OF A DUAL-FREQUENCY VECSEL AT $852 \mathrm{~nm}$ AND DISCUSSION}

Measuring the phase noise spectrum of a laser can be performed either by measuring the spectrum of its beatnote with another, if possible quieter, laser, or by performing a self-heterodyning experiment, or by converting the phase noise into an intensity noise through a resonant device such as a Fabry-Pérot resonator. Rather than performing such a difficult experiment, we take advantage of the fact that the motivation for this work lies in the discrepancy between a simpler model for thermal phase noise and measurements of the beatnote phase noise from a DF-VECSEL, ${ }^{9}$ as shown in Fig. 1(c). The high spectral purity of the RF beatnote generated with the DF-VECSEL dedicated to cesium CPT clocks is crucial for the clock frequency relative stability targeted by this metrology application. This is why, in Sec. IV, we compare the predictions of the preceding sections with the measurements previously reported for a 852-nm-DF-VECSEL. ${ }^{9}$ To this aim, we start by detailing how the beatnote phase noise is related to the optical phase noises of the two modes oscillating in the laser.

\section{A. Beatnote phase noise model}

We now consider the two orthogonally polarized laser modes produced by the DF-VECSEL described in Subsection II A. We deduce the phase noise of their beatnote from the preceding theoretical results. However, since the same laser pumps the two modes, one needs to take into account the correlations between the pump power fluctuations seen by these two modes. ${ }^{17}$ The modulus $\eta$ and the phase $\psi$ of this correlation have been shown to be to a large extent independent of the noise frequency in the range of frequencies that we consider here $(1 \mathrm{kHz}$ to $20 \mathrm{MHz})$. In these conditions, the PSD $S_{\phi_{T}}(f)$ of the beatnote phase noise induced by the pump fluctuations is related to the optical phase noise PSD $S_{\dot{\psi}}(f)$ through the following relation: ${ }^{17}$

$$
S_{\phi_{T}}(f)=\frac{S_{\dot{\psi}}(f)}{(2 \pi f)^{2}} \cdot \frac{P_{\mathrm{p}, x}^{2}+P_{\mathrm{p}, y}^{2}-2 \eta \cdot \cos \psi \cdot P_{\mathrm{p}, x} \cdot P_{\mathrm{p}, y}}{P_{\mathrm{opt}}^{2}},
$$

where $P_{\mathrm{p}, i}$ for $i=(x, y)$ stands for the optical pump power intercepted by each mode.

Since we suppose that the thermodynamic fluctuations seen by the two modes are independent, the part of the beatnote phase noise induced by thermodynamic fluctuations is directly given by

$$
S_{\phi_{T}}^{\text {Thermo }}(f)=2 S_{\dot{\psi}}^{\text {Thermo }}(f) /(2 \pi f)^{2} .
$$

\section{B. Comparison between experiment and theory}

Figure 7 reproduces the beatnote phase noise spectrum that we already reported ${ }^{9}$ for a DF-VECSEL oscillating at $852 \mathrm{~nm}$ (green filled circles), together with several theoretical spectra. The

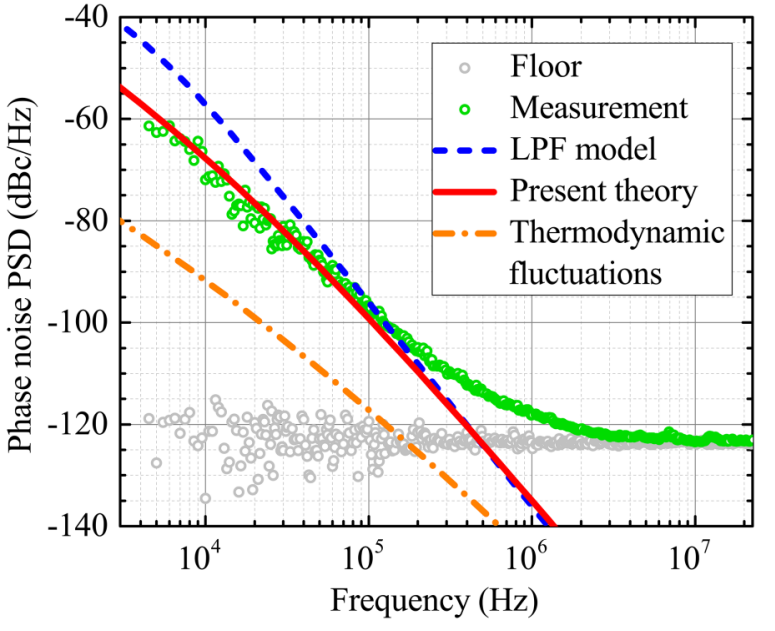

FIG. 7. RF beatnote phase noise PSD spectra for a dual-frequency VECSEL. The open green circles are already issued measurements, ${ }^{9}$ the open gray circles stand for the measurement floor, and the dashed blue line is the oversimplified low-pass filter (LPF) model for thermal noise. In the present theory of thermal noise, the contribution of the pump heat fluctuations is shown in the solid red line and one of the thermodynamic fluctuations is shown in the dashed-dotted orange line.

full red line and the orange dotted-dashed line were obtained using Eqs. (37) and (38), respectively, and thus correspond to the pump-induced and thermodynamic contributions to the phase noise, obtained with the parameters of the Appendix. Besides, the dashed blue line corresponds to the simple low-pass filter model. One should mention that in the frequency range considered here, thermal noises are the dominant contributions.'

These results clearly show that the theory developed here, based more particularly on Eqs. (37) and (30), is in excellent agreement with the experiment. In particular, the agreement is much better than with the simple low-pass filter model $^{15,18-21}$ that we used before to account for these measurements. ${ }^{9}$ Moreover, Fig. 7 shows that the noise induced by pump fluctuations is strongly dominant, by $20-30 \mathrm{~dB}$, over the one induced by thermodynamic fluctuations. However, at higher frequencies, in the range of a few megahertz, the thermodynamic fluctuations become as significant as the pump noise induced ones, although our measurement floor (open gray circles) does not allow us to check this experimentally.

\section{Validity of the low-pass filter model}

In the simple model we used previously, ${ }^{18}$ the frequency noise PSD was evaluated according to the expression

$$
S_{\dot{\psi}}(f)=\operatorname{RIN}_{\mathrm{p}}\left(\omega_{l} \cdot \Gamma_{T} \cdot R_{T}\right)^{2} /\left(1+(2 \pi f \tau)^{2}\right),
$$

with $R_{T}$ being the structure thermal resistance, $\Gamma_{T}$ is the relative thermal variation of the cavity optical length due to the refractive index variation with temperature, ${ }^{18}$ and $\tau$ is the typical diffusion time over the mode radius. 
This simple expression has several similarities with the more complete expression whose result in plotted in Fig. 5: according to Eq. (39), the frequency noise PSD is also white at low frequencies and exhibits a $f^{-2}$ roll-off at high frequencies. However, as can be noticed from Fig. 7 , the intermediate $f^{-3}$ behavior of the experimental phase noise PSD cannot be explained by such a model. Indeed, this would correspond to a $1 / f$ behavior for the frequency noise PSD. The limitations of the macroscopic approach of Eq. (39) come from the fact that it relies on a single characteristic time $\tau$ whereas in reality thermal diffusion occurs over both the pump width and the depth of the active medium, giving two different characteristic times, as we have seen in Subsection III D.

Moreover, we have seen above that the large number of terms in the infinite sum of Eq. (30) is responsible for the smoothing of the transition between $1 \mathrm{kHz}$ and $100 \mathrm{kHz}$ and is what makes the present model better fit the experimental slope in this frequency range.

\section{Discussion: Is an improved simplified model possible?}

The frequency noise PSDs calculated in Sec. IV have quite complicated expressions. Although the low-pass filter model was unable to fully account for the pump induced frequency noise, it was providing the good asymptotic behaviors. In this subsection, we thus wonder how to provide a simplified model that takes both transverse and longitudinal thermal diffusions into account. We try to perform this using asymptotic expressions of the present model.

\section{At low frequencies}

In the low-frequency range defined by $\omega \ll \omega_{1}$ with $\omega_{1}=\min \left[1 / \tau_{c}, \frac{\pi^{2} D_{/ /}}{4 L_{\mathrm{SC}}^{2}}\right]$, the thermal frequency fluctuations induced by the pump noise and described by Eq. (30) can be approximated as

$$
S_{\dot{\psi}}\left(\omega \ll \omega_{1}\right)=\operatorname{RIN}_{\mathrm{p}}(\omega) \cdot f_{0}^{2} \cdot\left|\sum_{n=0}^{\infty} \mathcal{N}_{n} \mathrm{e}^{a_{n} / 2} \mathrm{E}_{1}\left(\frac{a_{n}}{2}\right)\right|^{2},
$$

where $f_{0}$ is the thermal frequency shift due to the pump and defined as

$$
f_{0} \equiv \frac{q Q_{0} \pi w_{2}^{2} w_{\mathrm{p}}^{2} L_{\mathrm{SC}}}{8 D_{\perp} \mathcal{V}_{\text {mode }}}
$$

With our parameters (see the Appendix), the Fourier frequency $\omega_{1} / 2 \pi$ is typically related to the longitudinal diffusion time and is thus of the order of a few $100 \mathrm{~Hz}$. With a white pump intensity noise, Eq. (40) yields a constant value for the frequency noise PSD, which is the one observed below $100 \mathrm{~Hz}$ in the spectrum of Fig. 5.

In the low-frequency range given by $\omega \ll \omega_{2}$ with $\omega_{2}=\min \left[1 / \tau, \frac{\pi^{2} D_{/ /}}{4 L_{\mathrm{SC}}^{2}}\right]$, the thermodynamic contribution of
Eq. (35) simply reads

$$
S_{\dot{\psi}}^{\text {Thermo }}\left(\omega \ll \omega_{2}\right)=\sum_{n=0}^{\infty} \mathcal{M}_{n} \cdot \exp \left(b_{n}\right) \cdot \mathrm{E}_{1}\left(b_{n}\right)
$$

with

$$
\mathcal{M}_{n}=\frac{q^{2} \pi^{2} w_{2}^{4} k_{\mathrm{B}} T^{2}}{C_{v} \mathcal{V}_{\text {mode }}^{2} L_{\mathrm{SC}} D_{\perp}}\left(\int_{0}^{L_{\mathrm{SC}}} \mathrm{d} z \sin \left(\kappa_{n} z\right)\left|e_{/ /}(z)\right|^{2}\right)^{2} .
$$

In the present example, $\omega_{2}$ has the same value as $\omega_{1}$. The corresponding constant value of Eq. (42) can be observed below $1 \mathrm{kHz}$ in the spectrum of Fig. 6 .

\section{In the high-frequency limit}

In the high-frequency limit, an asymptotic expansion of the exponential integral function permits to express the pump thermally induced frequency fluctuations as

$$
S_{\dot{\psi}}\left(\omega \gg \omega_{1}\right)=\operatorname{RIN}_{\mathrm{p}}(\omega) \cdot f_{0}^{2} \cdot\left|\sum_{n=0}^{\infty} \frac{2 \mathcal{N}_{n}}{a_{n}+\mathrm{i} \omega \tau_{c}}\right|^{2} .
$$

Similarly, the thermodynamic contribution to the fluctuations reads

$$
S_{\dot{\psi}}^{\text {Thermo }}\left(\omega \gg \omega_{2}\right)=\sum_{n=0}^{\infty} \mathcal{M}_{n} \cdot \frac{b_{n}}{b_{n}^{2}+(\omega \tau)^{2}} .
$$

Both these expressions are compatible with the $f^{-2}$ asymptotic behaviors already mentioned but also with the smoothing of the spectrum corner induced by longitudinal diffusion.

\section{Interpolated approximation}

Based on these asymptotic expressions, we propose the following rational function series to approximate the two contributions to the thermal frequency noise:

$$
S_{\dot{\psi}}(\omega) \sim \operatorname{RIN}_{\mathrm{p}}(\omega) \cdot f_{0}^{2} \cdot\left|\sum_{n=0}^{\infty} \frac{\mathcal{N}_{n}}{\left(\mathrm{e}^{a_{n} / 2} \mathrm{E}_{1}\left(a_{n} / 2\right)\right)^{-1}+\mathrm{i} \omega \tau_{c} / 2}\right|^{2}
$$

and

$$
S_{\dot{\psi}}^{\text {Thermo }}(\omega) \sim \sum_{n=0}^{\infty} \mathcal{M}_{n} \cdot \frac{\left(\mathrm{e}^{b_{n}} \mathrm{E}_{1}\left(b_{n}\right)\right)^{-1}}{\left(\mathrm{e}^{b_{n}} \mathrm{E}_{1}\left(b_{n}\right)\right)^{-2}+(\omega \tau)^{2}} .
$$

Such expressions are easier to handle than the original equations in order to estimate and optimize the thermal frequency noise of a VECSEL.

\section{CONCLUSION}

In this paper, a complete model of the thermal frequency noise of an optically pumped VECSEL has been established, which is in 
TABLE I. Useful parameters for the implemented VECSEL structure.

\begin{tabular}{|c|c|c|c|c|c|}
\hline \multirow{2}{*}{ Parameter } & \multirow{2}{*}{ Symbol } & \multicolumn{3}{|c|}{ Value } & \multirow{2}{*}{ Units } \\
\hline & & $\mathrm{Al}_{22} \% \mathrm{GaAs}$ & & GaAs & \\
\hline $\begin{array}{l}\text { Specific heat per unit } \\
\text { of volume }\end{array}$ & $C_{v}$ & 1.77 & & 1.76 & $\mathrm{JK}^{-1} \mathrm{~cm}^{-3}$ \\
\hline Thermal diffusivity & $D$ & 0.11 & & 0.31 & $\mathrm{~cm}^{2} \mathrm{~s}^{-1}$ \\
\hline $\begin{array}{l}\text { Linear thermal } \\
\text { expansion coefficient }\end{array}$ & $\varsigma$ & 5.61 & & 5.73 & $\times 10^{-6} \mathrm{~K}^{-1}$ \\
\hline $\begin{array}{l}\text { Optical absorption } \\
\text { coefficient at } 673 \mathrm{~nm}\end{array}$ & $\alpha$ & 2 & & & $\times 10^{4} \mathrm{~cm}^{-1}$ \\
\hline $\begin{array}{l}\text { Refractive index at } \\
852 \mathrm{~nm}\end{array}$ & $n$ & 3.4 & & & \\
\hline $\begin{array}{l}\text { Refractive index at } \\
673 \mathrm{~nm}\end{array}$ & $n_{673}$ & 3.6 & & & \\
\hline Index thermal variation & $\partial n / \partial T$ & & & 4 & $\times 10^{-4} \mathrm{~K}^{-1}$ \\
\hline $\begin{array}{l}\text { Laser central } \\
\text { wavelength }\end{array}$ & $\lambda$ & & 852 & & $\mathrm{~nm}$ \\
\hline $\begin{array}{l}\text { Rescaled substrate } \\
\text { thickness }\end{array}$ & $L_{\mathrm{SUB}}$ & & 260 & & $\mu \mathrm{m}$ \\
\hline DBR thickness & $e_{\mathrm{DBR}}$ & & 4.1 & & $\mu \mathrm{m}$ \\
\hline DBR index step & $\Delta n$ & & 0.54 & & \\
\hline $\begin{array}{l}\text { Total thickness of } \\
\text { the chip }\end{array}$ & $L_{\mathrm{SC}}$ & & 266 & & $\mu \mathrm{m}$ \\
\hline Extended cavity length & $L_{\mathrm{CAV}}$ & & 5.07 & & $\mathrm{~cm}$ \\
\hline Optical pump power & $P_{\mathrm{opt}}$ & & 0.95 & & $\mathrm{~W}$ \\
\hline Pump incidence angle & $i_{1}$ & & $40^{\circ}$ & & \\
\hline $\begin{array}{l}\text { Output coupler } \\
\text { transmission }\end{array}$ & $T$ & & $0.5 \%$ & & \\
\hline Radius of pump mode & $w_{\mathrm{p}}$ & & 24 & & $\mu \mathrm{m}$ \\
\hline $\begin{array}{l}\text { Output coupler radius } \\
\text { of curvature }\end{array}$ & $R_{1}$ & & 5 & & $\mathrm{~cm}$ \\
\hline $\begin{array}{l}\text { Fraction of power } \\
\text { conversion }\end{array}$ & $\eta_{\text {th }}$ & & $20 \%$ & & \\
\hline $\begin{array}{l}\text { Pump relative intensity } \\
\text { noise }\end{array}$ & $\mathrm{RIN}_{\mathrm{p}}$ & & -130 & & $\mathrm{~dB} \mathrm{~Hz} z^{-1}$ \\
\hline $\begin{array}{l}\text { Longitudinal thermal } \\
\text { diffusivity }\end{array}$ & $D_{\| /}$ & & 0.23 & & $\mathrm{~cm}^{2} \mathrm{~s}^{-1}$ \\
\hline $\begin{array}{l}\text { Transverse thermal } \\
\text { diffusivity }\end{array}$ & $D_{\perp}$ & & 0.14 & & $\mathrm{~cm}^{2} \mathrm{~s}^{-1}$ \\
\hline Room temperature & $T$ & & 300 & & $\mathrm{~K}$ \\
\hline $\begin{array}{l}x \text {-polarization pump } \\
\text { power }\end{array}$ & $P_{\mathrm{p}, x}$ & & 0.70 & & $\mathrm{~W}$ \\
\hline $\begin{array}{l}y \text {-polarization pump } \\
\text { power }\end{array}$ & $P_{\mathrm{p}, y}$ & & 0.25 & & $\mathrm{~W}$ \\
\hline $\begin{array}{l}\text { Pump intensity } \\
\text { correlation amplitude }\end{array}$ & $\eta$ & & 0.98 & & \\
\hline
\end{tabular}

very good agreement with the measurements of the beatnote phase noise in a dual-frequency operation ${ }^{9}$ for metrology application.

First, the dynamics of heat diffusion coming from the optical pump source has shown to exhibit many different relevant time scales. The transient and steady-state regimes along the optical axis have been described by analytic expressions. The significant thermal lens effect induced by the pump has also been calculated. As a consequence, the laser beam radius in the semiconductor chip is strongly modified by the pump. Then, taking into account the pump intensity and thermodynamic fluctuations as well, analytic expressions were obtained for the frequency noise PSD induced via thermo-optic effects. The contribution of pump heating fluctuations to the phase noise measurements has been shown to be dominant at low frequencies. The discrepancy with respect to the previous oversimplified model has been shown to come from longitudinal diffusion, which is non-negligible considering the mode volume inside the active medium for the frequency range below $1 \mathrm{MHz}$. The present model involves longitudinal-to-transverse diffusion ratios in Fourier series expansions, which makes it more accurate to describe the physics of the thermal noise. Moreover, the anisotropy of heat diffusion has been included in the model, which is more suitable to describe multilayered structures such as VECSELs.

The generality of our theoretical predictions makes it applicable to other systems displaying a similar infinite plate geometry, such as modelocked integrated exernal-cavity surface-emitting laser used for dual-comb emission. ${ }^{38}$

\section{ACKNOWLEDGMENTS}

This work was supported by the Direction Générale de l'Armement and the Agence Nationale de la Recherche (CHOCOLA, No. ANR-15-CE24-0010-04; DIFOOL No. ANR15-ASMA-0007-04; LASAGNE, No. ANR-16-ASTR-0010-03) and performed in the framework of the joint lab between Laboratoire Aimé Cotton and Thales R\&T.

\section{APPENDIX: PARAMETERS}

All the parameters used in this article are gathered in Table I. It corresponds to the $1 / 2$-VCSEL structure based on AlGaAs compounds and the cavity that we have used in our preceding experiments.

\section{REFERENCES}

${ }^{1}$ V. Iakovlev, J. Walczak, M. Gẹbski, A. K. Sokół, M. Wasiak, P. Gallo, A. Sirbu, R. P. Sarzała, M. Dems, T. Czyszanowski, and E. Kapon, "Double-diamond high-contrast-gratings vertical external cavity surface emitting laser," J. Phys. D Appl. Phys. 47, 065104 (2014).

${ }^{2}$ N. Jornod, V. J. Wittwer, C. Kränkel, D. Waldburger, U. Keller, T. Südmeyer, and T. Calmano, "High-power amplification of a femtosecond vertical externalcavity surface-emitting laser in an YB:YAG waveguide," Opt. Express 25, 16527-16533 (2017).

${ }^{3}$ S. Knitter, C. Liu, B. Redding, M. A. Choma, and H. Cao, "Coherence switching of a degenerate VECSEL for multimodality imaging," in Conference on Lasers and Electro-Optics (Optical Society of America, 2016), p. AM3O.4.

${ }^{4}$ G. Baili, L. Morvan, M. Alouini, D. Dolfi, F. Bretenaker, I. Sagnes, and A. Garnache, "Experimental demonstration of a tunable dual-frequency semiconductor laser free of relaxation oscillations," Opt. Lett. 34, 3421-3423 (2009).

${ }^{\mathbf{5}}$ F. Bretenaker, S. De, F. Ihsan, F. Goldfarb, G. Baili, G. Pillet, L. Morvan, M. Alouini, A. El Amili, I. Sagnes, and S. Bouchoule, "Dual-frequency VECSELs for microwave photonics applications," in International Conference on Fibre Optics and Photonics (Optical Society of America, 2014).

${ }^{6}$ P. Dumont, F. Camargo, J.-M. Danet, D. Holleville, S. Guerandel, G. Pillet, G. Baili, L. Morvan, D. Dolfi, I. Gozhyk, G. Beaudoin, I. Sagnes, P. Georges, and G. Lucas-Leclin, "Low-noise dual-frequency laser for compact Cs atomic clocks," J. Lightwave Technol. 32, 3817-3823 (2014).

${ }^{7}$ P. D. D. Schwindt, S. Knappe, V. Shah, L. Hollberg, J. Kitching, L.-A. Liew, and J. Moreland, “Chip-scale atomic magnetometer," Appl. Phys. Lett. 85, 6409-6411 (2004). 
${ }^{8}$ J. Vanier, "Atomic clocks based on coherent population trapping: A review," Appl. Phys. B 81, 421-442 (2005).

${ }^{9}$ G. Gredat, D. Chatterjee, G. Baili, F. Gutty, I. Sagnes, F. Goldfarb, F. Bretenaker, and H. Liu, "Fully-correlated multi-mode pumping for low-noise dual-frequency VECSELs," Opt. Express 26, 26217-26226 (2018).

${ }^{10}$ T. Zanon, S. Guerandel, E. de Clercq, D. Holleville, N. Dimarcq, and A. Clairon, "High contrast Ramsey fringes with coherent-population-trapping pulses in a double lambda atomic system," Phys. Rev. Lett. 94, 193002 (2005).

${ }^{11}$ C. Henry, "Phase noise in semiconductor lasers," J. Lightwave Technol. 4, 298-311 (1986).

${ }^{12}$ A. E. Amili, G. Loas, S. De, S. Schwartz, G. Feugnet, J.-P. Pocholle, F. Bretenaker, and M. Alouini, "Experimental demonstration of a dual-frequency laser free from antiphase noise," Opt. Lett. 37, 4901-4903 (2012).

${ }^{13}$ S. De, A. E. Amili, I. Fsaifes, G. Pillet, G. Baili, F. Goldfarb, M. Alouini, I. Sagnes, and F. Bretenaker, "Phase noise of the radio frequency (RF) beatnote generated by a dual-frequency VECSEL," J. Lightwave Technol. 32, 1307-1316 (2014).

${ }^{14} \mathrm{~S}$. De, V. Potapchuk, and F. Bretenaker, "Influence of spin-dependent carrier dynamics on the properties of a dual-frequency vertical-external-cavity surface-emitting laser," Phys. Rev. A 90, 013841 (2014).

${ }^{15}$ S. De, G. Baili, M. Alouini, J.-C. Harmand, S. Bouchoule, and F. Bretenaker, "Class-A dual-frequency VECSEL at telecom wavelength," Opt. Lett. 39, 5586-5589 (2014).

${ }^{16}$ S. De, G. Baili, S. Bouchoule, M. Alouini, and F. Bretenaker, "Intensity- and phase-noise correlations in a dual-frequency vertical-external-cavity surfaceemitting laser operating at telecom wavelength," Phys. Rev. A 91, 053828 (2015).

${ }^{17}$ H. Liu, G. Gredat, S. De, I. Fsaifes, A. Ly, R. Vatré, G. Baili, S. Bouchoule, F. Goldfarb, and F. Bretenaker, "Ultra-low noise dual-frequency VECSEL at telecom wavelength using fully correlated pumping," Opt. Lett. 43, 1794-1797 (2018).

${ }^{18}$ H. Liu, G. Gredat, G. Baili, F. Gutty, F. Goldfarb, I. Sagnes, and F. Bretenaker, "Noise investigation of a dual-frequency VECSEL for application to cesium clocks," J. Lightwave Technol. 36, 3882-3891 (2018).

${ }^{19}$ A. Laurain, M. Myara, G. Beaudoin, I. Sagnes, and A. Garnache, "High power single-frequency continuously-tunable compact extended-cavity semiconductor laser," Opt. Express 17, 9503-9508 (2009).

${ }^{20}$ A. Laurain, M. Myara, G. Beaudoin, I. Sagnes, and A. Garnache, "Multiwatt-power highly-coherent compact single-frequency tunable verticalexternal-cavity-surface-emitting-semiconductor-laser," Opt. Express 18, $14627-14636$ (2010).

${ }^{21}$ A. Laurain, L. Cerutti, M. Myara, and A. Garnache, "2.7- $\mu \mathrm{m}$ single-frequency $\mathrm{TEM}_{00}$ low-threshold Sb-based diode-pumped external-cavity VCSEL,” IEEE Photonics Technol. Lett. 24, 246-248 (2012).
${ }^{22}$ Y. G. Zhao and J. G. McInerney, "Transient temperature response of verticalcavity surface-emitting semiconductor lasers," IEEE J. Quantum Electron. 31, 1668-1673 (1995).

${ }^{23}$ D. I. Babic and S. W. Corzine, "Analytic expressions for the reflection delay, penetration depth, and absorptance of quarter-wave dielectric mirrors," IEEE J. Quantum Electron. 28, 514-524 (1992).

${ }^{24}$ F. Abelès and P. Baumeister, "Multilayer reflectors with minimal dispersion of differential phase shift upon reflection," Opt. Commun. 93, 1-3 (1992).

${ }^{25}$ Y. Huo, C. Y. Cho, K. F. Huang, Y. F. Chen, and C. C. Lee, "Exploring the DBR superlattice effect on the thermal performance of a VECSEL with the finite element method," Opt. Lett. 44, 327-330 (2019).

${ }^{26} \mathrm{G}$. Chen, C. Tien, X. Wu, and J. Smith, "Thermal diffusivity measurement of GaAs/AlGaAs thin-film structures," J. Heat Transfer 116, 325-331 (1994).

${ }^{27}$ B. Diu, C. Guthmann, D. Lederer, and B. Roulet, Physique Statistique (Hermann Paris, 1989).

${ }^{28} \mathrm{~K}$. M. van Vliet, "Markov approach to density fluctuations due to transport and scattering. II. Applications," J. Math. Phys. 12, 1998-2012 (1971).

${ }^{29} \mathrm{~K}$. M. van Vliet, "Markov approach to density fluctuations due to transport and scattering. I. Mathematical formalism," J. Math. Phys. 12, 1981-1998 (1971).

${ }^{30} \mathrm{~K}$. M. Van Vliet and H. Mehta, "Theory of transport noise in semiconductors," Phys. Status Solidi B 106, 11-30 (1981).

${ }^{31}$ C. Lauer and M.-C. Amann, "Calculation of the linewidth broadening in vertical-cavity surface-emitting lasers due to temperature fluctuations," Appl. Phys. Lett. 86, 191108 (2005)

${ }^{32}$ S. Foster, A. Tikhomirov, and M. Milnes, "Fundamental thermal noise in distributed feedback fiber lasers," IEEE J. Quantum Electron. 43, 378-384 (2007).

${ }^{33}$ S. Foster, "Fundamental limits on $1 / f$ frequency noise in rare-earth-metaldoped fiber lasers due to spontaneous emission," Phys. Rev. A 78, 013820 (2008).

${ }^{34}$ L. Landau and E. Lifshitz, Course of Theoretical Physics, VI: Fluid Mechanics (MIR Editions, 1971)

${ }^{35}$ M. Leutenegger, "Matlab file exchange: Hankel transform," see https://fr. mathworks.com/matlabcentral/fileexchange/13371-hankel-transform (2007).

${ }^{36}$ M. E. Innocenzi, H. T. Yura, C. L. Fincher, and R. A. Fields, "Thermal modeling of continuous-wave end-pumped solid-state lasers," Appl. Phys. Lett. 56, 1831-1833 (1990).

${ }^{37}$ W. Koechner and M. Bass, Solid-State Lasers: A Graduate Text (Springer Science \& Business Media, 2006).

${ }^{38}$ S. M. Link, D. J. H. C. Maas, D. Waldburger, and U. Keller, "Dual-comb spectroscopy of water vapor with a free-running semiconductor disk laser," Science 356, 1164-1168 (2017) 